\title{
One-pot synthesis of highly functionalised $1 H$-pyrazoles from arylcarbohydrazides, cyclohexyl isocyanide, and acetylene diesters
}

\author{
Mohammad Anary-Abbasinejad, ${ }^{* a}$ Nasim Shams ${ }^{b}$ and Motahareh Heidari \\ ${ }^{a}$ Department of Chemistry, Rafsanjan Vali-e-Asr University, Rafsanjan, PO Box 77176, Iran \\ ${ }^{b}$ Department of Chemistry, Islamic Azad University, Yazd Branch, P.O. Box 89195-155, Yazd, \\ Iran
}

E-mail: mohammadanary@yahoo.com

\begin{abstract}
A novel one-pot isocyanide-based cascade four-component reaction between arylcarbohydrazides, dialkyl acetylenedicarboxylates, and cyclohexyl isocyanide lead to dialkyl 1-(1,2-bisalkoxycarbonylvinyl)-5-cyclohexylamino-2-aroyl-2,3-dihydro-1 $H$-pyrazole-3,4-dicarboxylates in excellent yields.
\end{abstract}

Keywords: Dialkyl acetylenedicarboxylates, cyclohexyl isocyanide, multi-component reactions, $1 H$-pyrazoles, arylcarbohydrazides

\section{Introduction}

Multi-component reactions (MCRs) are important for generating high levels of diversity, as they allow more than two building blocks to be combined in a practical, time-saving, one-pot operation, giving rise to complex structures by simultaneous formation of two or more bonds. ${ }^{1}$ As a special subclass, the isocyanide-based MCRs (IMCRs) offer a number of advantages originating from the unique reactivity of an isocyanide, which acts as a nucleophile and an electrophile at the same time. MCRs contribute to the requirements of an environmentally friendly process by reducing the number of synthetic steps, energy consumption, and waste production. ${ }^{2}$ MCRs, which lead to interesting heterocyclic scaffolds, are particularly useful for the construction of diverse chemical libraries of "drug-like" molecules. Furthermore, the discovery of novel MCRs can be considered as an interesting topic for academic research, which also satisfies a practical interest of applied science. ${ }^{3}$

Compounds containing pyrazole ring are extensively used in pharmaceutical, agrochemical, food and cosmetic industries as well as being used as complexing agents for the synthesis of hydrogenation catalysts and UV stabilizers. ${ }^{4-8}$ Moreover, pyrazolines and pyrazoles play a 
crucial role in the development of theory in heterocyclic chemistry and also are extensively used as useful synthons in organic synthesis. ${ }^{9}$

As part of our ongoing program to develop efficient and robust methods for the preparation of heterocyclic compounds, ${ }^{10-13}$ herein we wish to report an efficient and simple approach to the synthesis of dialkyl 1-[1,2-bis(alkoxycarbonyl)vinyl]-5-cyclohexylamino-2-aroyl-2,3-dihydro$1 H$-pyrazole-3,4-dicarboxylates by reaction between arylcarbohydrazides, dialkyl acetylenedicarboxylates and cyclohexyl isocyanide in good yields.

\section{Results and Discussion}

Reaction between furyl-2-carbohydrazide (1a), dimethyl acetylenedicarboxylate (DMAD, 2a, 2 eq.) and cyclohexyl isocyanide after $24 \mathrm{~h}$ stirring in dichloromethane at ambient temperature and column chromatography afforded dimethyl 1-[1,2-bis(methoxycarbonyl)vinyl]-5-cyclohexylamino-2-(furan-2-yl)-2,3-dihydro-1 $H$-pyrazole-3,4-dicarboxylate (5a) in 98\% yield (Scheme 1).<smiles>[R]C(=O)NN</smiles>

1a: $R=2$-furyl 1b: $R=$ phenyl<smiles>[R]OC(=O)C#CC(=O)O[R2]</smiles>

2a: $\mathrm{R}^{\prime}=\mathrm{Me}$

2b: $\mathrm{R}^{\prime}=\mathrm{Et}$

2c: $\mathrm{R}^{\prime}=t-\mathrm{Bu}$<smiles>[R]OC(=O)C#CC(=O)O[R2]</smiles>

3a: $\mathrm{R}^{\prime \prime}=\mathrm{Me}$

3b: R" = Et

3c: $\mathrm{R}^{\prime \prime}=t-\mathrm{Bu}$

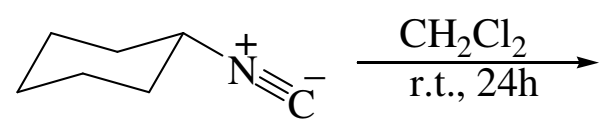

4

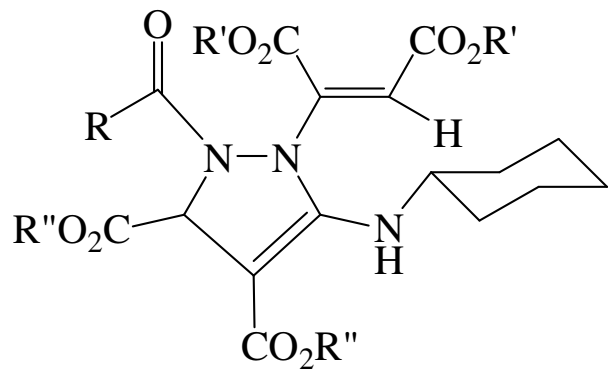

5

\begin{tabular}{|c|c|c|c|c|}
\hline 5 & $\mathrm{R}$ & $\mathrm{R}^{\prime}$ & $\mathrm{R}^{\prime \prime}$ & Yield of $\mathbf{5}^{*}$ \\
\hline $\mathbf{a}$ & 2-Furyl & $\mathrm{Me}$ & $\mathrm{Me}$ & 98 \\
\hline b & 2-furyl & $\mathrm{Et}$ & $\mathrm{Et}$ & 95 \\
\hline c & 2-furyl & $t-\mathrm{Bu}$ & $t-\mathrm{Bu}$ & 90 \\
\hline d & phenyl & $\mathrm{Me}$ & $\mathrm{Me}$ & 96 \\
\hline 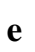 & phenyl & Et & $\mathrm{Et}$ & 94 \\
\hline f & phenyl & $t-\mathrm{Bu}$ & $t-\mathrm{Bu}$ & 90 \\
\hline g & phenyl & $\mathrm{Me}$ & $\mathrm{Et}$ & 89 \\
\hline h & phenyl & $\mathrm{Me}$ & $\mathrm{t}-\mathrm{Bu}$ & 85 \\
\hline
\end{tabular}

Scheme 1. Reaction between arylcarbohydrazides, acetylenic diesters, and cyclohexyl isocyanide. 
The structures of the products were deduced from their IR, mass, ${ }^{1} \mathrm{H}$ NMR, and ${ }^{13} \mathrm{C}$ NMR spectra. The IR spectrum of compound 5a exhibited an absorption band at $3290 \mathrm{~cm}^{-1}$ for the NH group and three strong broad absorption bands at 1680, 1718, $1745 \mathrm{~cm}^{-1}$ for carbonyl groups. The mass spectrum of this compound displayed molecular ion peak at the appropriate $m / z$ value. The ${ }^{1} \mathrm{H}$ NMR spectrum of compound 5a consisted of multiplet signals for the methylene groups of cyclohexyl ring at $\delta=1.05-1.67 \mathrm{ppm}$ and a multiplet signal at $\delta=3.98 \mathrm{ppm}$ for the $\mathrm{CH}$ of cyclohexyl ring. Four singlet signals were observed for methoxy groups at $\delta=3.61,3.62,3.68$ and $3.70 \mathrm{ppm}$. A broad doublet at $\delta=6.47 \mathrm{ppm}$ was observed for the NH proton, disappeared by the addition of $\mathrm{D}_{2} \mathrm{O}$ to $\mathrm{CDCl}_{3}$ solution of 5a. The proton of the methine group and the olefinic proton were observed as two singlet signals at $\delta=5.52$ and $5.75 \mathrm{ppm}$, respectively. The protons of the furan ring showed signals at $\delta=6.46,7.30$, and $7.53 \mathrm{ppm} .{ }^{13} \mathrm{C}$ NMR spectrum of compound 5a showed 24 distinct resonances in agreement with the proposed structure, partial assignments of these resonances are given in the experimental section. The NMR data for compounds 5a-h shows the presence of only one isomer, but with the available NMR data we could not definitely establish the configuration.

To explore the scope and limitations of this reaction further, we extended our studies to the benzohydrazide and other dialkyl acetylenedicarboxylates such as diethyl- and di-t-butyl acetylenedicarboxylate. As indicated in Scheme 1, the reactions proceeded efficiently to produce compounds (5b-f).



Scheme 2. Suggested mechanism for formation of compounds 5 . 
We also examined the one-pot reaction between two different dialkyl acetylenedicarboxylates with phenylcarbohydrazide and cyclohexyl isocyanide. Thus, diethyl acetylenedicarboxylate (DEAD, 3b) or di-t-butyl acetylenedicarboxylate (DTAD, 3c) and cyclohexyl isocyanide were successively added to a mixture of DMAD and phenylcarbohydrazide in dichloromethane. After stirring for $24 \mathrm{~h}$ at room temperature and column chromatography, the desired products $\mathbf{5} \mathbf{g}$ and $\mathbf{5 h}$ were obtained in good yields, respectively.

A mechanistic rationalization for this reaction is provided in Scheme 2. The Michael addition of arylcarbohydrazide $\mathbf{1}$ to acetylene diester $\mathbf{2}$ affords the adduct $\mathbf{6}$. The zwitterion $\mathbf{7}$, produced by the addition of cyclohexyl isocyanide to acetylene diester $\mathbf{3}$, is porotonated by compound 6 to yield the nitrilium cation 8. Michael addition of the conjugate base of 6 (anion 9) to nitrilium cation $\mathbf{8}$ leads to ketenimine $\mathbf{1 0}$, which then cyclizes to product $\mathbf{5}$.

\section{Conclusions}

In summary, we report herein a new and efficient method for the synthesis of highly functionalized $1 H$-pyrazole derivatives by a four-component reaction between arylcarbohydrazides, dialkyl acetylenedicarboxylates, and cyclohexyl isocyanide. Due to the easy availability of the synthetic approach and the neutral ring closure conditions, this new synthetic approach discussed here has the potential in synthesis of various functionalized $1 H$ pyrazole derivatives. The reactions carry the advantages that the starting materials are simply available and may be used without any purification or modification under neutral conditions.

\section{Experimental Section}

General. All melting points are uncorrected. Elemental analyses were performed using a Heraeus CHN-O-Rapid analyzer. Mass spectra were recorded on a Finnigan-MAT 8430 mass spectrometer operating at an ionization potential of $70 \mathrm{eV}$. IR spectra were recorded on a Shimadzu IR-470 spectrometer. ${ }^{1} \mathrm{H}$, and ${ }^{13} \mathrm{C}$ NMR spectra were recorded on Bruker DRX-500 Avance spectrometer at 500.1 and $125.8 \mathrm{MHz}$, respectively. ${ }^{1} \mathrm{H}$, and ${ }^{13} \mathrm{C} \mathrm{NMR}$ spectra were obtained on solution in $\mathrm{CDCl}_{3}$ using TMS as internal standard. Column chromatography was performed with Merck silica gel 60, 230-400 mesh. The chemicals used in this work purchased from Fluka (Buchs, Switzerland) and were used without further purification.

General synthetic procedure for compounds 5a-f, exemplified by dimethyl 1-(1,2-bis(methoxycarbonylvinyl)-5-cyclohexylamino-2-(2-furyl)-2,3-dihydro-1H-pyrazole-3,4-dicarboxylate (5a)

To a stirred solution of (furan-2-yl)carbohydrazide $\mathbf{1}\left(\begin{array}{lll}1 & \mathrm{mmol}\end{array}\right)$ and dimethyl acetylenedicarboxylate $2(2 \mathrm{mmol})$ in $\mathrm{CH}_{2} \mathrm{Cl}_{2}(10 \mathrm{~mL})$ was added drop-wise cyclohexyl 
isocyanide $4(1 \mathrm{mmol})$ in $\mathrm{CH}_{2} \mathrm{Cl}_{2}(2 \mathrm{~mL})$ at room temperature over $10 \mathrm{~min}$. The reaction mixture was stirred at room temperature. After completion of the reaction $(24 \mathrm{~h})$ as indicated by TLC (AcOEt/hexane, 1:1), the solvent was removed under reduced pressure and the residue was purified by column chromatography over silica gel (Merck 60 mesh) using a 2:1 hexane-AcOEt mixture as eluent to afford the pure product.

5a. Yellow powder, yield: $98 \%$; $\mathrm{mp} 164-166^{\circ} \mathrm{C}$, IR (KBr) $\left(v_{\max }, \mathrm{cm}^{-1}\right): 3290(\mathrm{NH}), 1680,1718$, $1745(\mathrm{C}=\mathrm{O})$. MS (m/z, \%): $519\left(\mathrm{M}^{+}, 7\right) .{ }^{1} \mathrm{H}$ NMR (500 $\left.\mathrm{MH}_{\mathrm{Z}}, \mathrm{CDCl}_{3}\right): 1.05-1.67$ (10 H, m, 5 $\left.\mathrm{CH}_{2}\right), 3.98$ (1 H, m, CH), 3.61, 3.62, 3.68 and $3.70\left(12 \mathrm{H}, 4 \mathrm{~s}, 4 \mathrm{OCH}_{3}\right), 6.47(1 \mathrm{H}$, broad d, NH), 5.52, 5.75 (2 H, 2s, pyrazole and olefinic CH's), $6.46\left(1 \mathrm{H}, \mathrm{dd},{ }^{3} J_{\mathrm{HH}} 3.5 \mathrm{~Hz},{ }^{3} J_{\mathrm{HH}} 1.7 \mathrm{~Hz}, \mathrm{CH}\right.$ furan), $7.30\left(1 \mathrm{H}, \mathrm{d},{ }^{3} J_{\mathrm{HH}} 3.5 \mathrm{~Hz}, \mathrm{CH}\right.$ furan), $7.53\left(1 \mathrm{H}, \mathrm{d},{ }^{3} J_{\mathrm{HH}} 1.7 \mathrm{~Hz}, \mathrm{CH}\right.$ furan $) .{ }^{13} \mathrm{C}$ NMR $\left(125.7 \mathrm{MHz}, \mathrm{CDCl}_{3}\right): 24.5,24.9,25.6,32.6$ and $34.7\left(5 \mathrm{CH}_{2}\right), 51.5,52.2,53.1$ and 53.4 (4 $\left.\mathrm{OCH}_{3}\right), 55.6(\mathrm{CH}-\mathrm{NH}), 64.1\left(\mathrm{CH}-\mathrm{CO}_{2} \mathrm{Me}\right), 88.0,106.2,112.1,120.3,144.5,147.6,150.4$ and 158.8 (aromatic and olefinic carbons), 162.6, 163.1, 165.8, 169.1 and 169.6 (5 C=O). Analysis: Calcd. for $\mathrm{C}_{24} \mathrm{H}_{29} \mathrm{~N}_{3} \mathrm{O}_{10}$ : C, 55.49; H, 5.63; N, 8.09. Found: C, 55.35; H, 5.80; N, 7.92\%.

Diethyl 1-[1,2-bis(ethoxycarbonyl)vinyl]-5-cyclohexylamino-2-(2-furyl)-2,3-dihydro-1Hpyrazole-3,4-dicarboxylate (5b). Yellow powder, yield: $95 \%$; mp 167-169 ${ }^{\circ} \mathrm{C}$, IR $(\mathrm{KBr})\left(v_{\max }\right.$, $\left.\mathrm{cm}^{-1}\right): 3280(\mathrm{NH}), 1675,1720,1750(\mathrm{C}=\mathrm{O}) . \mathrm{MS}(\mathrm{m} / \mathrm{z}, \%): 575(\mathrm{M} .+, 9) .{ }^{1} \mathrm{H}$ NMR $\left(500 \mathrm{MH}_{\mathrm{Z}}\right.$, $\left.\mathrm{CDCl}_{3}\right): \delta 1.03-1.71\left(10 \mathrm{H}, \mathrm{m}, 5 \mathrm{CH}_{2}\right), 1.16,1.23,1.34$ and $1.45\left(12 \mathrm{H}, 4 \mathrm{t},{ }^{3} \mathrm{~J}_{\mathrm{HH}} 7 \mathrm{~Hz}, 4 \mathrm{CH}_{3}\right)$, $3.94(1 \mathrm{H}, \mathrm{m}, \mathrm{CH}), 4.12,4.19,4.27$ and $4.35\left(8 \mathrm{H}, 4 \mathrm{q},{ }^{3} J_{\mathrm{HH}} 7 \mathrm{~Hz}, 4 \mathrm{OCH}_{2}\right), 5.53$ and $5.73(2 \mathrm{H}$, 2 s, pyrazole and olefinic CH's), $6.51\left(1 \mathrm{H}\right.$, broad d, NH), $6.47\left(1 \mathrm{H}, \mathrm{dd},{ }^{3} J_{\mathrm{HH}} 3.5 \mathrm{~Hz},{ }^{3} J_{\mathrm{HH}} 1.7\right.$ $\mathrm{Hz}, \mathrm{CH}), 7.30\left(1 \mathrm{H}, \mathrm{d}^{3} J_{\mathrm{HH}} 3.5 \mathrm{~Hz}\right), 7.53\left(1 \mathrm{H}, \mathrm{d}^{3} J_{\mathrm{HH}} 1.7 \mathrm{~Hz}, \mathrm{CH}\right.$ furan). ${ }^{13} \mathrm{C}$ NMR (125.7 $\left.\mathrm{MHz}, \mathrm{CDCl}_{3}\right): \delta 13.0,13.2,13.4$ and $13.5\left(4 \mathrm{CH}_{3}\right), 24.5,24.7,25.3,32.5$ and $34.7\left(5 \mathrm{CH}_{2}\right), 56.6$ $(\mathrm{CH}-\mathrm{NH}), 60.6,60.9,61.8$ and $62.6\left(4 \mathrm{OCH}_{2}\right), 64.1\left(\mathrm{CH}-\mathrm{CO}_{2} \mathrm{Me}\right), 88.4,108.2,110.5,124.3$, 140.6, 145.1, 152.3 and 158.9 (aromatic and olefinic carbons), 162.5, 162.7, 163.4, 168.8 and 170.5 (5 C=O). Analyses: Calcd. for $\mathrm{C}_{28} \mathrm{H}_{37} \mathrm{~N}_{3} \mathrm{O}_{10}$ : C, 58.43; H, 6.48; N, 7.30. Found: C, 58.49; $\mathrm{H}, 6.22 ; \mathrm{N}, 7.41 \%$.

Di-t-butyl 1-[1,2-bis(t-butoxycarbonyl)vinyl]-5-cyclohexylamino-2-(2-furyl)-2,3-dihydro$1 \boldsymbol{H}$-pyrazole-3,4-dicarboxylate (5c). Yellow powder, yield: $90 \%$; mp 172-174 ${ }^{\circ} \mathrm{C}$, IR (KBr) $\left(v_{\max }, \mathrm{cm}-1\right): 3290(\mathrm{NH}), 1685,1724,1745(\mathrm{C}=\mathrm{O})$. MS $(\mathrm{m} / \mathrm{z}, \%): 687\left(\mathrm{M}^{+}, 6\right) .{ }^{1} \mathrm{H}$ NMR $(500$ $\left.\mathrm{MHz}, \mathrm{CDCl}_{3}\right): \delta 1.05-1.67\left(10 \mathrm{H}, \mathrm{m}, 5 \mathrm{CH}_{2}\right), 1.39,1.42,1.50$ and $1.54(36 \mathrm{H}, 4 \mathrm{~s}, 4 t-\mathrm{Bu}), 3.98(1$ $\mathrm{H}, \mathrm{m}, \mathrm{CH}), 5.53$ and 5.75 (2 H, $2 \mathrm{~d}$, pyrazole and olefinic CH's), 6.47 (1 H, broad d, NH), 6.46 $\left(1 \mathrm{H}, \mathrm{dd},{ }^{3} J_{\mathrm{HH}} 3.5 \mathrm{~Hz},{ }^{3} J_{\mathrm{HH}} 1.7 \mathrm{~Hz}, \mathrm{CH}\right), 7.30\left(1 \mathrm{H}, \mathrm{d},{ }^{3} J_{\mathrm{HH}} 3.5 \mathrm{~Hz}\right), 7.53\left(1 \mathrm{H}, \mathrm{d},{ }^{3} J_{\mathrm{HH}} 1.7\right.$ $\mathrm{Hz}, \mathrm{CH}$ furan). ${ }^{13} \mathrm{C} \mathrm{NMR}\left(125.7 \mathrm{MHz}, \mathrm{CDCl}_{3}\right): \delta 24.6,24.8,25.2,32.3$ and $34.2\left(5 \mathrm{CH}_{2}\right), 26.9$, 27.1, 27.8 and $28.0\left(12 \mathrm{CH}_{3}\right.$ of $\left.4 t-\mathrm{Bu}\right), 55.2(\mathrm{CH}-\mathrm{NH}), 65.1\left(\mathrm{CH}-\mathrm{CO}_{2} \mathrm{Me}\right), 81.2,81.3,81.5$ and 81.6 (4 C of $4 t-\mathrm{Bu}$ ), 89.2, 105.4, 111.5, 122.7, 142.3, 147.8, 151.1 and 158.7 (aromatic and olefinic carbons), 162.4, 162.5, 163.4, 168.9 and 170.1 (5 $\mathrm{C}=\mathrm{O})$. Analyses: Calcd. for $\mathrm{C}_{36} \mathrm{H}_{53} \mathrm{~N}_{3} \mathrm{O}_{10}$ : C, 62.86; H, 7.77; N, 6.11. Found: C, 62.70; H, 7.90; N, 6.21\%.

Dimethyl 1-[1,2-bis(methoxycarbonyl)vinyl]-5-cyclohexylamino-2-benzoyl-2,3-dihydro-1Hpyrazole-3,4-dicarboxylate (5d). Yellow powder, yield: $96 \%$; mp 152-154 ${ }^{\circ} \mathrm{C}$, IR (KBr) $\left(v_{\max }\right.$, $\left.\mathrm{cm}^{-1}\right): 3320(\mathrm{NH}), 1680,1722,1740(\mathrm{C}=\mathrm{O}) . \mathrm{MS}(\mathrm{m} / \mathrm{z}, \%): 529\left(\mathrm{M}^{+}, 7\right) .{ }^{1} \mathrm{H}$ NMR $(500 \mathrm{MHz}$, 
$\left.\mathrm{CDCl}_{3}\right): \delta$ 1.05-1.67 $\left(10 \mathrm{H}, \mathrm{m}, 5 \mathrm{CH}_{2}\right), 3.62,3.65,3.67$ and $3.71\left(12 \mathrm{H}, 4 \mathrm{~s}, 4 \mathrm{OCH}_{3}\right), 3.95(1 \mathrm{H}$, $\mathrm{m}, \mathrm{CH}), 5.54$ and $5.76(2 \mathrm{H}, 2 \mathrm{~d}$, pyrazole and olefinic $\mathrm{CH}$ 's), 6.48 (1 H, broad d, NH), 7.26-7.59 (5 H, aromatic). ${ }^{13} \mathrm{C}$ NMR (125.7 MHz, $\left.\mathrm{CDCl}_{3}\right): \delta 24.4,24.7,25.6,32.4$ and $34.8\left(5 \mathrm{CH}_{2}\right), 52.0$, 52.3, 52.5 and $52.7\left(4 \mathrm{OCH}_{3}\right), 55.5(\mathrm{CH}-\mathrm{NH}), 64.2\left(\mathrm{CH}-\mathrm{CO}_{2} \mathrm{Me}\right), 88.1,107.4,127.3,127.7$, 128.3, 133.0, 146.2, and 158.6 (aromatic and olefinic carbons), 162.1, 162.4, 164.4, 168.9 and 169.9 (5 C=O). Analyses: Calcd. for $\mathrm{C}_{26} \mathrm{H}_{31} \mathrm{~N}_{3} \mathrm{O}_{9}$ : C, 58.97; H, 5.90; N, 7.94. Found: C, 58.78; $\mathrm{H}, 5.80 ; \mathrm{N}, 8.02 \%$.

Diethyl 1-[1,2-bis(ethoxycarbonyl)vinyl]-5-cyclohexylamino-2-benzoyl-2,3-dihydro-1Hpyrazole-3,4-dicarboxylate (5e). Yellow powder, yield: $94 \%$; mp $155-157{ }^{\circ} \mathrm{C}$, IR $(\mathrm{KBr})\left(v_{\max }\right.$, $\left.\mathrm{cm}^{-1}\right): 3290(\mathrm{NH}), 1685,1718,1747(\mathrm{C}=\mathrm{O}) . \mathrm{MS}(\mathrm{m} / \mathrm{z}, \%): 585\left(\mathrm{M}^{+}, 11\right) .{ }^{1} \mathrm{H}$ NMR $(500 \mathrm{MHz}$, $\left.\mathrm{CDCl}_{3}\right): \delta 1.01-1.70\left(10 \mathrm{H}, \mathrm{m}, 5 \mathrm{CH}_{2}\right), 1.15,1.20,1.32$ and $1.45\left(12 \mathrm{H}, 4 \mathrm{t},{ }^{3} J_{\mathrm{HH}} 7 \mathrm{~Hz}, 4 \mathrm{CH}_{3}\right)$, $3.92(1 \mathrm{H}, \mathrm{m}, \mathrm{CH}), 4.11,4.18,4.29$ and $4.34\left(8 \mathrm{H}, 4 \mathrm{q},{ }^{3} \mathrm{~J}_{\mathrm{HH}} 7 \mathrm{~Hz}, 4 \mathrm{OCH}_{2}\right), 5.51$ and $5.73(2 \mathrm{H}$, 2 s, pyrazole and olefinic CH's), $6.53\left(1 \mathrm{H}\right.$, broad d, NH), 7.24-7.58 (5 H, aromatic). ${ }^{13} \mathrm{C}$ NMR $\left(125.7 \mathrm{MHz}, \mathrm{CDCl}_{3}\right): \delta 13.1,13.3,13.6$ and $13.7\left(4 \mathrm{CH}_{3}\right), 24.0,24.1,25.9,32.5$ and 34.8 (5 $\left.\mathrm{CH}_{2}\right), 56.3(\mathrm{CH}-\mathrm{NH}), 60.5,60.9,61.7$ and $62.5\left(4 \mathrm{OCH}_{2}\right), 64.3\left(\mathrm{CH}-\mathrm{CO}_{2} \mathrm{Me}\right), 88.3,105.2,126.9$, $127.5,128.1,134.0,145.5$, and 158.2 (aromatic and olefinic carbons), 161.7, 162.1, 164.7, 168.9 and $169.8(5 \mathrm{C}=\mathrm{O})$. Analyses: Calcd. for $\mathrm{C}_{30} \mathrm{H}_{39} \mathrm{~N}_{3} \mathrm{O}_{9}$ : C, 61.53; H, 6.71; N, 7.18. Found: $\mathrm{C}$, $61.70 ; \mathrm{H}, 6.55 ; \mathrm{N}, 7.26 \%$.

Di-t-butyl 1-[1,2-bis(t-butoxycarbonyl)vinyl]-5-cyclohexylamino-2-benzoyl-2,3-dihydro-1Hpyrazole-3,4-dicarboxylate (5f). Yellow powder, yield: $90 \%$; mp 160-162 ${ }^{\circ} \mathrm{C}, \mathrm{IR}(\mathrm{KBr})\left(v_{\max }\right.$, $\left.\mathrm{cm}^{-1}\right)$ : $3290(\mathrm{NH}), 1682,1728,1740(\mathrm{C}=\mathrm{O})$. MS (m/z, \%): $697\left(\mathrm{M}^{+}, 9\right) .{ }^{1} \mathrm{H}$ NMR (500 MHz, $\left.\mathrm{CDCl}_{3}\right): \delta 1.05-1.68\left(10 \mathrm{H}, \mathrm{m}, 5 \mathrm{CH}_{2}\right), 1.37,1.41,1.49$ and $1.53(36 \mathrm{H}, 4 \mathrm{~s}, 4 t-\mathrm{Bu}), 3.97(1 \mathrm{H}, \mathrm{m}$, $\mathrm{CH}), 5.51$ and 5.74 (2 H, 2 d, pyrazole and olefinic CH's), 6.47 (1 H, broad d, NH), 7.23-7.57 (5 $\mathrm{H}$, aromatic). $\left.{ }^{13} \mathrm{C} \mathrm{NMR} \mathrm{(125.7} \mathrm{MHz}, \mathrm{CDCl}_{3}\right): \delta 24.2,24.7,25.7,32.6$ and $34.9\left(5 \mathrm{CH}_{2}\right), 26.9$, 27.1, 27.8 and $28.0\left(12 \mathrm{CH}_{3}\right.$ of $4 t$-Bu $), 55.2(\mathrm{CH}-\mathrm{NH}), 65.1\left(\mathrm{CH}-\mathrm{CO}_{2} \mathrm{Me}\right), 81.0,81.2,81.5$ and 81.6 (4 C of $4 t$-Bu), 88.3, 106.1, 126.9, $127.7,128.3,134.1,144.5$, and 159.1 (aromatic and olefinic carbons), 161.0, 162.2, 164.7, 169.1 and 170.8 (5 $\mathrm{C}=\mathrm{O})$. Analyses: Calcd. for $\mathrm{C}_{38} \mathrm{H}_{55} \mathrm{~N}_{3} \mathrm{O}_{9}$ : C, 65.40; H, 7.94; N, 6.02. Found: C, 65.59; H, 7.82; N, 6.14\%.

Preparation of compound $(\mathbf{5 g})$ and $(5 \mathrm{~h})$. A solution of arylcarbohydrazide 1 (1 mmol) and dialkyl acetylenedicarboxylate $2(1 \mathrm{mmol})$ in $\mathrm{CH}_{2} \mathrm{Cl}_{2}(10 \mathrm{~mL})$ was stirred for $5 \mathrm{~min}$. Dialkyl acetylenedicarboxylate 3 (1 mmol) was added to above mixture and then a mixture of cyclohexyl isocyanide $4(1 \mathrm{mmol})$ in $\mathrm{CH}_{2} \mathrm{Cl}_{2}(2 \mathrm{~mL})$ was added drop-wise at room temperature over $10 \mathrm{~min}$. The rest of the process is similar to that for compounds $\mathbf{5 a - f .}$

Diethyl 1-[1,2-bis(methoxycarbonyl)vinyl]-5-cyclohexylamino-2-benzoyl-2,3-dihydro-1Hpyrazole-3,4-dicarboxylate (5g). Yellow powder, yield: $89 \%$; mp 143-145 ${ }^{\circ} \mathrm{C}, \mathrm{IR}(\mathrm{KBr})\left(v_{\max }\right.$, $\left.\mathrm{cm}^{-1}\right): 3290(\mathrm{NH}), 1678,1718,1750(\mathrm{C}=\mathrm{O})$. MS (m/z, \%): $557\left(\mathrm{M}^{+}, 12\right) .{ }^{1} \mathrm{H}$ NMR $(500 \mathrm{MHz}$, $\left.\mathrm{CDCl}_{3}\right): \delta$ 1.05-1.67 $\left(10 \mathrm{H}, \mathrm{m}, 5 \mathrm{CH}_{2}\right), 1.26$ and $1.30\left(6 \mathrm{H}, 2 \mathrm{t},{ }^{3} \mathrm{~J}_{\mathrm{HH}} 7 \mathrm{~Hz}, 2 \mathrm{CH}_{3}\right), 3.61$ and 3.70 $\left(6 \mathrm{H}, 2 \mathrm{~s}, 2 \mathrm{OCH}_{3}\right), 3.98(1 \mathrm{H}, \mathrm{m}, \mathrm{CH}), 4.20$ and $4.24\left(4 \mathrm{H}, 2 \mathrm{q},{ }^{3} J_{\mathrm{HH}} 7 \mathrm{~Hz}, 2 \mathrm{OCH}_{2}\right), 5.25$ and 5.75 (2 H, 2 s, pyrazole and olefinic CH's), 6.47 (1H, broad d, NH), 7.23-7.58 (5 H, aromatic). ${ }^{13} \mathrm{C} \mathrm{NMR}\left(125.7 \mathrm{MHz}, \mathrm{CDCl}_{3}\right): \delta 14.4$ and $15.6\left(2 \mathrm{CH}_{3}\right), 24.6,24.7,25.6,28.3$ and $34.8\left(5 \mathrm{CH}_{2}\right)$, 
52.1 and $52.8\left(2 \mathrm{OCH}_{3}\right), 55.6(\mathrm{CH}-\mathrm{NH}), 61.6$ and $62.1\left(2 \mathrm{OCH}_{2}\right), 64.1\left(\mathrm{CH}-\mathrm{CO}_{2} \mathrm{Me}\right), 88.0$, 105.3, 126.9, 127.5, 128.1, 134.0, 145.1, and 158 (aromatic and olefinic carbons), 161.8, 162.3, 165.9, 168.7 and 169.4 (5 C=O). Analyses: Calcd. for $\mathrm{C}_{28} \mathrm{H}_{35} \mathrm{~N}_{3} \mathrm{O}_{9}$ : C, 60.31; H, 6.33; N, 7.54. Found: C, 60.15; H, 6.25; N, 7.66\%.

Di-t-butyl 1-[1,2-bis(methoxycarbonyl)vinyl]-5-cyclohexylamino-2-benzoyl-2,3-dihydro-1Hpyrazole-3,4-dicarboxylate (5h). Yellow powder, yield: $85 \%$; mp 146-148 ${ }^{\circ} \mathrm{C}$, IR $(\mathrm{KBr})\left(v_{\max }\right.$, $\left.\mathrm{cm}^{-1}\right): 3290(\mathrm{NH}), 1680,1724,1751(\mathrm{C}=\mathrm{O})$. MS (m/z, \%): $613\left(\mathrm{M}^{+}, 6\right) .{ }^{1} \mathrm{H}$ NMR (500 MHz, $\left.\mathrm{CDCl}_{3}\right): \delta 1.05-1.67\left(10 \mathrm{H}, \mathrm{m}, 5 \mathrm{CH}_{2}\right), 1.38$ and $1.50(18 \mathrm{H}, 2 \mathrm{~s}, 2 t-\mathrm{Bu}), 3.61$ and $3.70(6 \mathrm{H}, 2 \mathrm{~s}$, $\left.2 \mathrm{OCH}_{3}\right), 3.98(1 \mathrm{H}, \mathrm{m}, \mathrm{CH}), 6.47(1 \mathrm{H}$, broad d, NH), 5.23 and $5.74(2 \mathrm{H}, 2 \mathrm{~s}$, pyrazole and olefinic CH's), 7.25-7.59 (5 H, aromatic). $\left.{ }^{13} \mathrm{C} \mathrm{NMR} \mathrm{(125.7} \mathrm{MHz}, \mathrm{CDCl}_{3}\right): \delta 28.7$ and 28.8 (6 $\mathrm{CH}_{3}$ of $2 t$-Bu), 24.5, 24.6, 25.6, 28.3 and $34.7\left(5 \mathrm{CH}_{2}\right), 52.1$ and $52.4\left(2 \mathrm{OCH}_{3}\right), 55.7(\mathrm{CH}-\mathrm{NH})$, $64.3\left(\mathrm{CH}-\mathrm{CO}_{2} \mathrm{Me}\right), 81.2$ and $82.4(2 \mathrm{C}$ of $2 t$ - $\mathrm{Bu}), 88.5,106.2,126.9,127.7,128.3,134.3,144.2$, and 159.1 (aromatic and olefinic carbons), 161.6, 162.1, 165.6, 168.8 and 169.6 (5 C=O). Analyses: Calcd. for $\mathrm{C}_{32} \mathrm{H}_{43} \mathrm{~N}_{3} \mathrm{O}_{9}$ : C, 62.63; H, 7.06; N, 6.85. Found: C, 62.84; H, 7.21; N, $6.64 \%$.

\section{References}

1. (a) Bagley, M. C.; Dale, J. W. J. Chem. Commun. 2002, 1682. (b) Mont, N.; Teixid, J.; Borrell, J. I.; Kappe, C. O. Tetrahedron Lett. 2003, 44, 5385. (c) Simon, C.; Constantieux, T.; Rodriguez, J. Eur. J. Org. Chem. 2004, 24, 4957. (d) Cui, S. L.; Lin, X. F.; Wang, Y. G. J. Org. Chem. 2005, 70, 2866. (e) Huang, Y. J.; Yang, F. Y.; Zhu, C. J. J. Am. Chem. Soc. 2005, 127, 16387. (f) Ramn, D. J.; Yus, M. Angew. Chem., Int. Ed. 2005, 44, 1602.

2. (a) Dömling, A. Chem. Rev. 2006, 106, 17. (b) Dömling, A.; Ugi, I. Angew. Chem., Int. Ed. 2000, 39, 3168.

3. Orru, R. V. A.; Greef, M. Synthesis 2003, 1471. (b) Zhu, J.; Bienayme, H. Multicomponent Reactions; Wiley-VCH: Weinheim, 2005.

4. Murineddu, G.; Ruiu, S.; Mussinu, J.; Loriga, G.; Grella, G. E.; Carai, M.; Lazzari, P.; Pani, L.; Pinna, G. A. Bioorg. Med. Chem. 2005, 13, 3309.

5. Bhat, L.; Jandeleit, B.; Dias, T. M. ; Moors, T. L.; Gallop, M. A. Bioorg. Med. Chem. Lett. 2005, 15,85 .

6. Sridhar, R.; Perumal, P. T.; Etti, S.; Shanmugam, G.; Ponnuswamy, M. N.; Prabavathy, V. R.; Mathivanan, N. Bioorg. Med. Chem. Lett. 2004, 14, 6035.

7. Bekhit, A. A.; Abdel-Aziem, T. Bioorg. Med. Chem. 2004, 12, 1935.

8. Bhat, B.A.; Dhar, K. L.; Puri, S. C.; Saxena, A. K.; Shanmugavel, M.; Qazi, G. N.; Bioorg. Med. Chem. Lett. 2005, 15, 3177.

9. Padmavathi,V.; Sumathi, R. P.; Babu, N. C.; Reddy, D. B. J. Chem. Res. (S) 1999, 610.

10. Anary-Abbasinejad, M.; Mosslemin, M. H.; Anaraki-Ardakani, H. Synlett 2009, 16, 2676.

11. Anary-Abbasinejad, M.; Poorhassan,E.; Hassanabadi, A. Synlett 2009, 12,1929. 
12. Anary-Abbasinejad, M.; Charkhati, K.; Anaraki-Ardakani, H. Synlett 2009, 7, 1115.

13. Anary-Abbasinejad, M.; Mazraeh-Seffid, M.; Poorhassan, E.; Hassanabadi, A.; Rastegari, F. Arkivoc 2008 (xvii) 265.

14. Matter, U. E., Pascual, C., Pretsch, E., Pross, A., Simon, W., Sternhell, S. Tetrahedron, 1969, 25, 691 . 